Annales Geophysicae (2002) 20: 1265-1277 (C) European Geophysical Society 2002

\title{
Estimating random transverse velocities in the fast solar wind from EISCAT Interplanetary Scintillation measurements
}

\author{
A. Canals ${ }^{1}$, A. R. Breen ${ }^{1}$, L. Ofman ${ }^{2}$, P. J. Moran ${ }^{1}$, and R. A. Fallows ${ }^{1}$ \\ ${ }^{1}$ Physics Department, University of Wales, Aberystwyth, Wales, UK \\ ${ }^{2}$ The Catholic University of America and NASA Goddard Space Flight Center, Greenbelt, MD 20771, USA
}

Received: 17 October 2001 - Revised: 21 February 2002 - Accepted: 13 March 2002

\begin{abstract}
Interplanetary scintillation measurements can yield estimates of a large number of solar wind parameters, including bulk flow speed, variation in bulk velocity along the observing path through the solar wind and random variation in transverse velocity. This last parameter is of particular interest, as it can indicate the flux of low-frequency Alfvén waves, and the dissipation of these waves has been proposed as an acceleration mechanism for the fast solar wind. Analysis of IPS data is, however, a significantly unresolved problem and a variety of a priori assumptions must be made in interpreting the data. Furthermore, the results may be affected by the physical structure of the radio source and by variations in the solar wind along the scintillation ray path. We have used observations of simple point-like radio sources made with EISCAT between 1994 and 1998 to obtain estimates of random transverse velocity in the fast solar wind. The results obtained with various a priori assumptions made in the analysis are compared, and we hope thereby to be able to provide some indication of the reliability of our estimates of random transverse velocity and the variation of this parameter with distance from the Sun.
\end{abstract}

Key words. Interplanetary physics (MHD waves and turbulence; solar wind plasma; instruments and techniques)

\section{Introduction}

For many years observations of Interplanetary Scintillation (IPS) - the fluctuation in the radio power received from a compact radio source when the line of sight passes close to the Sun - have been used to calculate the velocity of the solar wind (e.g. Dennison and Hewish, 1967; Hewish et al., 1964; Bourgois et al., 1985; Kojima and Kakinuma, 1990; Rickett and Coles, 1991). Density irregularities in the solar wind cause variations in the refractive index, which gives rise to phase variations in the radio waves from compact sources. As these waves continue through space, the normal

Correspondence to: A. R. Breen (azb@aber.ac.uk) process of constructive and destructive interference change these phase variations into amplitude variations. As the irregularities move out in the solar wind, the effect is to cast a drifting diffraction pattern across the Earth. If the pattern is sampled using two radio telescopes when the ray paths from the radio source to the antenna lie in the same radial plane a plane passing through the center of the Sun - then a high degree of correlation is present between the scintillation at the two sites. The time lag for maximum correlation provides a direct estimation of the scintillation drift velocity. If the ray paths are sufficiently far from the Sun for the differences in phase changes introduced by the irregularities to be small, when measured over the Fresnel scale - the condition of weak scattering - then the contributions from different regions of the ray path combine as a simple weighted linear sum, making it possible to distinguish contributions from different regions of the ray path (Coles, 1996).

In situ measurements have established that near to solar minimum the solar wind has two distinct components: a fast stream flowing at about 750 to $800 \mathrm{~km} / \mathrm{s}$ and a slow stream with a flow speed of about 350 to $400 \mathrm{~km} / \mathrm{s}$ (e.g. Schwenn, 1990; Phillips et al., 1994; Woch et al., 1997; McComas et al., 1998, 2000). The fast wind originates from open magnetic field regions (coronal holes), which are conspicuous as dark regions in maps of coronal white-light intensity (Snyder and Neugebauer, 1966; Krieger and Timothy, 1973; Neupert and Pizzo, 1974; Nolte et al., 1976; Coles et al., 1980), although recently, there have been suggestions that fast wind may also emanate from the "quiet Sun" regions adjacent to the coronal holes (Habbal and Woo, 2001). This identification of fast streams of solar wind with outflow from coronal holes was one of the early successes of IPS (e.g. Coles et al., 1980). The slow wind is found predominantly above the streamer belts which have relatively high densities (Habbal et al., 1997; Woo and Martin, 1997) and so they can be associated with bright regions in white-light maps, though the exact source of the slow wind has not yet been fully established. The clear two-component distribution of the solar wind has been confirmed in IPS data (e.g. Breen et al., 1996b). Given 
the clear association between large regions of dark corona (coronal holes) and high-speed streams, this bimodal distribution makes it possible to use white-light intensity in coronal maps (a) to determine whether a given region of an IPS ray path is immersed in a fast or slow flow and thus - in the weak scattering regime - (b) to separate the contributions to the observed scattering pattern of the fast and slow winds (Coles, 1996; Grall et al., 1996; Breen et al., 1996b, 1998). In this paper, we consider observations that are overwhelmingly dominated by fast wind.

By comparing the auto-and cross-spectra of the scintillation patterns observed at the two receiving sites with the results of a 2-dimensional weak scattering model (Grall, 1995; Coles, 1996; Klinglesmith 1997; Grall et al., 1997; Massey, 1998), it is possible to obtain accurate estimates not only of the bulk flow speed, but also of secondary parameters, such as systematic variation of the flow speed across different regions of the ray path and random velocity perpendicular to the radial direction (e.g. Grall, 1995; Klinglesmith, 1997). This technique enables the derivation of the solar wind at all heliocentric latitudes, as well as over a wide range of distances from the Sun, from inside 10 solar radii (R) to beyond the Earth's orbit (Coles, 1995). However, since the method requires weak scattering, in order to cover the whole range of solar distances from the corona out to beyond $1 \mathrm{AU}(215 \mathrm{R})$, it is necessary to use a corresponding range of observing frequencies, with measurements closer to the Sun requiring higher frequencies. In this study, we use data taken from the EISCAT UHF facility. This system consists of three sites, at Ramfjordmoen (near Troms $\varnothing$, in Norway), Kiruna (Sweden) and Sodankylä (Finland), receiving over a 8-MHz bandwidth (5 MHz from spring 1999) centered on 931.5 MHz. The EISCAT system was constructed as an ionospheric radar, and is described in this role by Rishbeth and Williams (1985), but its low-noise receivers, high timing accuracy and long baselines between sites make it a very powerful facility for IPS observations, as described by Bourgois et al. (1985) and Breen et al. (1996a, b; 1998). The first IPS observations at EISCAT were made in 1982 and regular, comprehensive campaigns have taken place every year during the arctic summer (April-October) since 1990 (Coles et al., 1991). EISCAT observations of the low-density fast wind are clearly in the weak scattering regime outside 20-22 R (Fallows, 2001; Fallows et al., 2002b), and reliable bulk velocities can be determined out to $\sim 100 \mathrm{R}$ for stronger radio sources.

The first studies of random variation in solar wind velocity were carried out by R. D. Ekers and L. T. Little in 1971, with the theoretical method published in Little and Ekers (1971) and the first results of this analysis reported in Ekers and Little (1971). The Little and Ekers (1971) method was adopted for the interpretation of EISCAT data by Bourgois et al. (1985). The possibility of using these estimates of random transverse velocities to provide an upper limit to the Alfvén wave flux was recognized by Grall (1995) and the results were presented by Klinglesmith (1997).

The acceleration of the fast solar wind is one of the key problems of solar physics. IPS observations have indicated that the fast wind is accelerated rapidly, reaching its cruising velocity inside 10-15 R (Grall et al., 1996; Breen et al., 2000, 2002b), suggesting a close link between the mechanisms heating the corona and accelerating the solar wind. The dissipation of high-frequency Alfvén waves by ion-cyclotron resonance has been suggested as the mechanism responsible for coronal heating and solar wind acceleration on open field lines (Axford and McKenzie, 1997; Liewer et al., 1999), and this is supported by recent SOHO UVCS observations which show an increase in ion temperature with ion mass and perpendicular temperatures greater than temperatures parallel to the magnetic field (Kohl et al., 1997, 1998). Objections have been raised to the direct heating of the corona by highfrequency Alfvén waves, since modelling suggests that a substantial proportion of a high-frequency wave flux launched from the coronal base would be absorbed well below the observed temperature peak (Cranmer, 2000) - although debate continues on this issue (Tu and Marsch, 2001). Ofman and Davila $(1997,1998)$ have suggested an alternative model in which large amplitude low-frequency Alfvén waves could transport energy from the coronal base to the acceleration region of the fast solar wind, then dissipate via nonlinear interactions, thereby heating the corona and driving the outflow of the fast solar wind.

Alfvén waves are characterized by oscillating perturbations of the transverse magnetic field. The effect that the Alfvén wave has on the plasma travelling radially outwards from the Sun is to introduce a perpendicular component to the bulk flow of the solar wind. Hence, measurements of the random transverse velocity can provide an indication of how the Alfvén waves are dissipating as they travel from the Sun, and a study of this dissipation can provide insight into whether Alfvén waves are a key mechanism in the acceleration of the solar wind. IPS observations can, therefore, in principle, place an upper bound on the transverse velocity introduced by large amplitude, low-frequency Alfvén waves and, therefore, be used to test models of solar wind acceleration which predict dissipation of these waves. They cannot, however, be used to test models which rely on direct heating of the corona by high-frequency Alfvén waves, since these introduce transverse perturbations on scales which are too small to be detectable.

\section{IPS analysis}

The diffraction pattern observed at the Earth is caused by scattering along the whole line of sight. In weak scattering, the model sums the scattering from each point along the line of sight, giving each contribution a weighting that is roughly proportional to $R^{-4}$, where $R$ is the distance from the Sun. The weighting assumes a uniformly expanding solar atmosphere and such a rapid drop-off with distance means that the majority of scattering is in the region where $R$ is at a minimum, i.e. the point of closest approach to the Sun (e.g. Grall, 1995; Coles, 1996; Klinglesmith, 1997; Massey, 1998). IPS arises from variations in the refractive index of the solar wind 
and, therefore, the variance in the square of the electron density $\sigma^{2} N_{e}^{2}$; to a first approximation, $\sigma^{2} N_{e}^{2} \propto N_{e}^{2}$. In a constant velocity, spherically-diverging system, $N_{e} \propto R^{-2}$ so $\sigma^{2} N_{e}^{2} \propto R^{-4}$ and the ends of the line of sight (far distant from the point of closest approach to the Sun - the "Ppoint") contribute relatively little scintillation. The region of the ray path, which contributes $50 \%$ of the observed scintillation, subtends slightly less than $40^{\circ}$ at the Sun in a uniform spherically-expanding solar wind (38०: Grall, 1995) and more than $70 \%$ of the observed scintillation comes from that part of the ray path which subtends $70^{\circ}$ centered on the P-point.

In strong scattering the phase changes are large and the scattered waves interfere with both the scattered and incident waves. It is no longer possible to assume that the final scattering pattern is a linear sum of all the scattering events. Analysis of IPS data for the study presented in this paper was carried out using the 2-D weak scattering analysis model developed at UCSD. The main purpose of this analysis program is to make estimates of solar wind velocities. However, it can also yield values for other parameters in the solar wind, such as random velocities perpendicular to the mean flow and the systematic variation of the bulk flow speed across different regions of the ray path. The model assumes weak scattering and works by fitting a theoretical auto- and cross-spectra to the observed data, using a nonlinear least-squares minimisation with varying solar wind parameters, to reach a best fit to the data spectrum. Therefore, it allows for the analysis of observations which have two distinct solar wind velocities (fast and slow), yielding estimates for fast and slow solar wind velocities. The analysis assumes that the solar wind across the ray path consists of no more than two components, either "all fast", "all slow" or a region of fast flow flanked by regions of slow flow. The boundaries of the fast and slow streams are determined by mapping back the IPS ray path back onto synoptic maps, so that solar wind originating from coronal holes crossing the ray path can be identified. Two angles, $\theta_{\text {in }}$ and $\theta_{\text {out }}$, measured at the center of the Sun and defined relative to the normal, to the ray path at the point of closest approach to the Sun, describe which parts of the IPS line of sight have contributions from fast and slow wind. $\theta_{\text {in }}$ is measured from the point of closest approach toward the Earth in (negative) degrees, while $\theta_{\text {out }}$ is measured toward the source in (positive) degrees. Figure 1 shows the angles $\theta_{\text {in }}$ and $\theta_{\text {out }}$ in the case when fast flow is flanked by slow flow on either side.

The variation in the bulk outflow speed over each section of the ray path and the random transverse velocity can also be estimated. Although the model fits the data in the frequency domain, the method of determination can be more clearly visualized by considering the time domain. In the following figures, the dashed line shows the auto- and crosscorrelation functions predicted by the 2-D weak scattering model, while the solid line shows the corresponding functions calculated from the spectra of the scintillations received at the two antennas. Random transverse velocities and the systematic variation in the bulk outflow speed (in the parallel direction) both have an effect on the cross-correlation func-

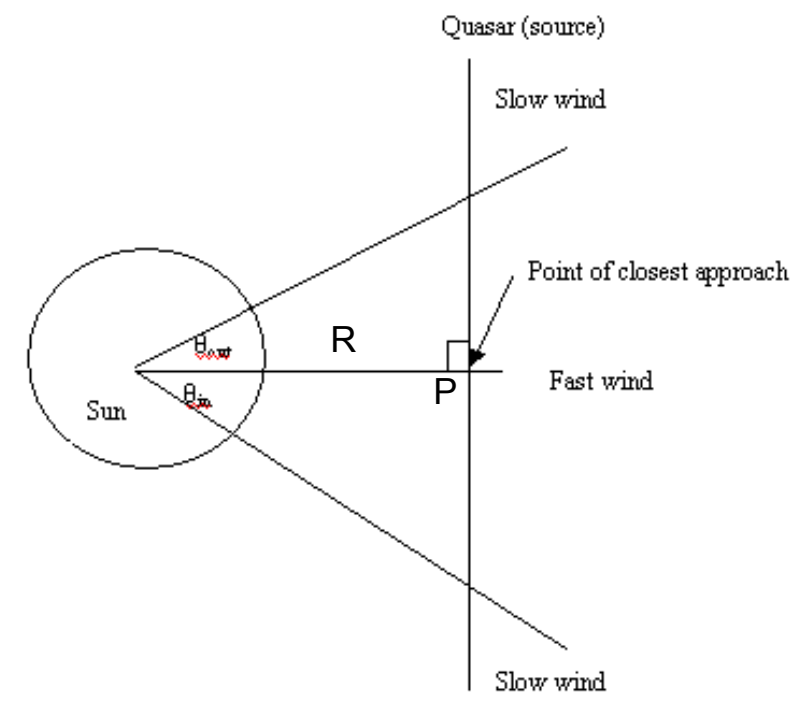

Earth

Fig. 1. Schematic diagram of the geometry of an IPS observation, shown as if looking down on the North Pole of the Sun. The ray paths from radio source to receiver pass through the extended solar atmosphere, with their point of closest approach to the Sun $(P)$ lying at a distance of $R$ solar radii from the center of the Sun. $\theta_{\text {in }}$ and $\theta_{\text {out }}$ define the angular extent of the ray path which is immersed in fast solar wind: in the coordinate system adopted in this study, angles are measured from the Sun-P direction and are positive towards the radio source, and negative towards the Earth.

tion. These velocities change the diffraction pattern between the two observing sites, which leads to a de-correlation of the cross-correlation function. Increasing values of random transverse velocities (RMS- $V_{\perp}$ ) and systematic variations in bulk flow $\left(d V_{\|}\right)$both de-correlate the function, but have different effects on the shape and skew of the function. Figure $2 \mathrm{a}$ shows the effect of increasing RMS- $V_{\perp}$ on the correlation function. As RMS- $V_{\perp}$ increases, the height of the cross-correlation function decreases, but the overall shape of the function remains substantially unchanged, with the whole function moving to shorter time lags. In Fig. $2 b$, the changes in the auto- and cross-correlation functions as $d V_{\|}$are illustrated. A small degree of de-correlation is introduced, but the most marked change is the substantial degree of skew towards shorter time lags which develops as $d V_{\|}$is increased. These results should be compared with the effects observed by Bourgois et al. (1985) when fitting IPS observations with smaller separations between the two sites.

Klinglesmith (1997) assumed that any velocities perpendicular to the bulk flow direction in the fast wind were introduced by Alfvén waves. This made it possible to use the estimates of RMS- $V_{\perp}$ provided by the IPS analysis program to give an upper bound for the Alfvén wave amplitude as a function of heliocentric distance. Figure $3 \mathrm{a}$ shows the results for RMS- $V_{\perp}$ (referred to as $d V_{\perp}$ in the figure) as a dotted line, and compares them to the expected variation in a spherically-expanding solar wind with constant velocity, in 


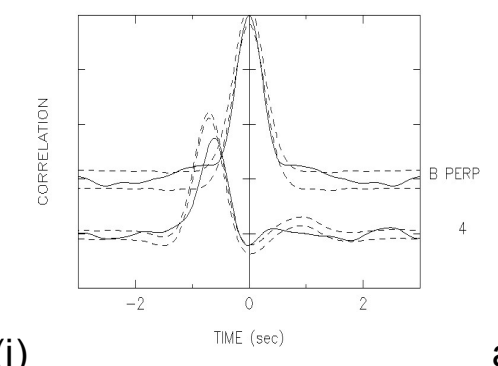

a(i)

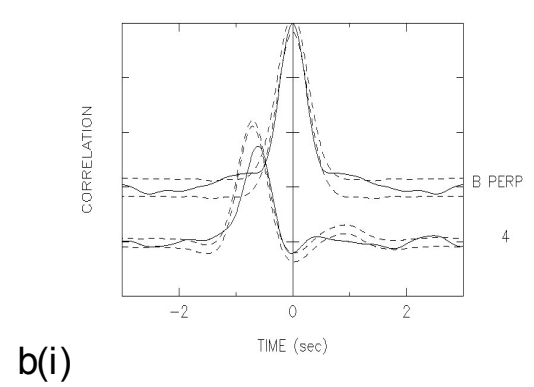

a(ii)

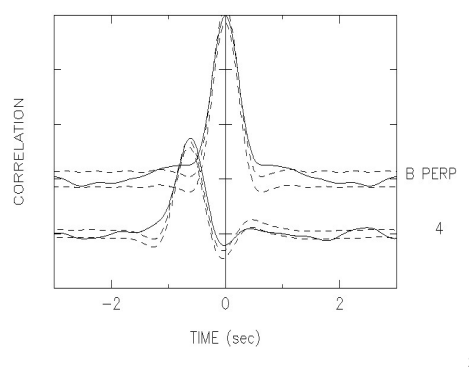

a(iii)
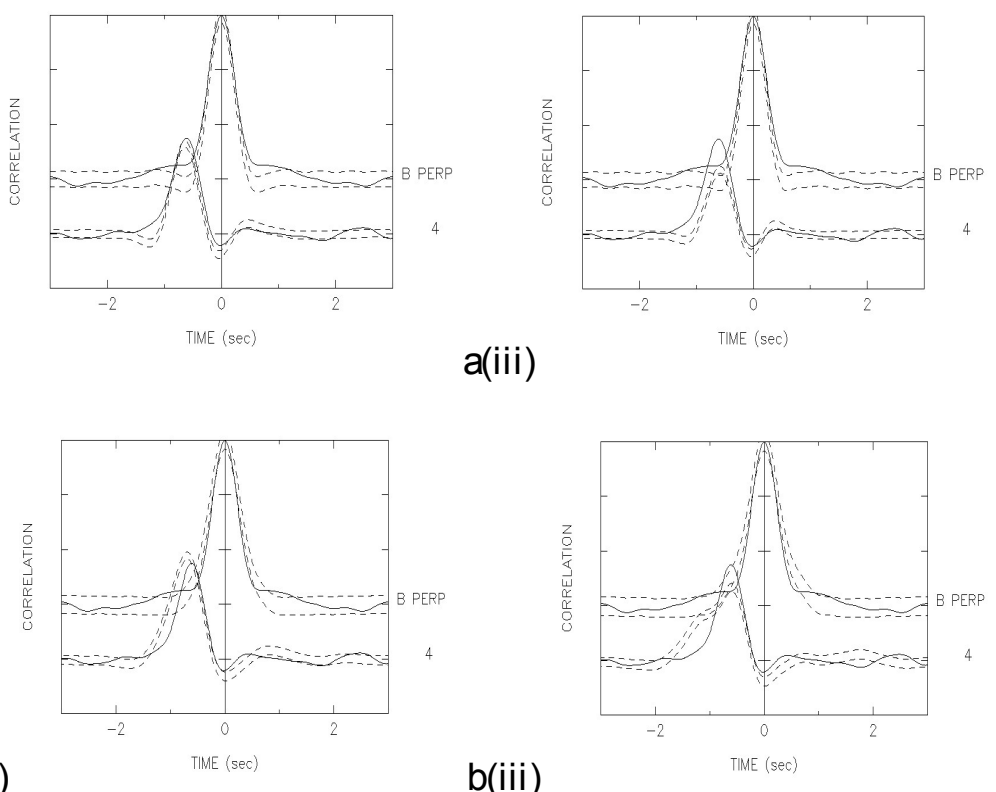

b(ii)

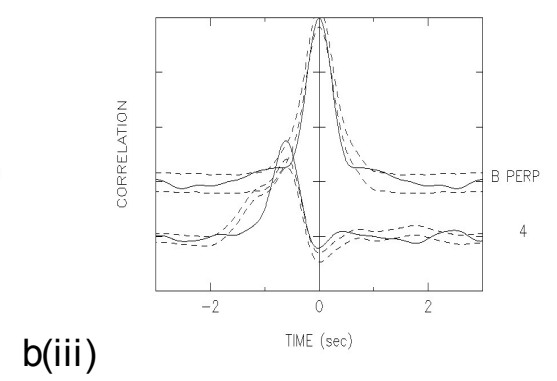

Fig. 2. The effects of increasing (a) random transverse velocity and (b) the variation in outflow velocity across the ray path on the modelled IPS auto- and cross-correlation functions. In all cases, the model is the dashed line and the data fitted are from an observation of $0431+$ 206 on 4 May 1999. In (a) the random transverse velocity, RMS- $V_{\perp}$, is set to $0 \mathrm{~km} / \mathrm{s}$ in (i), $1200 \mathrm{~km} / \mathrm{s}$ in (ii) and $1800 \mathrm{~km} / \mathrm{s}$ in (iii). The shape of the cross-correlation function does not change significantly, but the peak becomes lower and the whole function moves to shorter time lags. In (b) the variation in outflow velocity across the ray path, $d V_{\|}$, is set to $0 \mathrm{~km} / \mathrm{s}$ in (i), $80 \mathrm{~km} / \mathrm{s}$ in (ii) and $160 \mathrm{~km} / \mathrm{s}$ in (iii). The cross-correlation function becomes de-correlated and skewed slightly towards zero time lag. The shape of the function becomes broader.

which the relation

$$
\frac{\delta V}{\delta V_{0}}=2\left(\sqrt{R / R_{0}}+\sqrt{R_{0} / R}\right)^{-1}
$$

is valid, i.e. a constant velocity, spherically-expanding solar wind which is adequately described by the WKB approximation. $\delta V$ is the Alfvén wave velocity displacement at a distance $R$, and $\delta V_{0}$ is the wave velocity displacement at the Alfvén critical point $R_{0}$, taken to be at $12 R$, where the fast solar wind is equal to the local Alfvén speed. The variation of Alfvén wave amplitude expected in this case is shown as a solid line in Fig. 3a, with $V_{\text {Alvén }}=750 \mathrm{~km} / \mathrm{s}$, $\delta V_{0}=75 \mathrm{~km} / \mathrm{s}$ and $R_{0}=12$ solar radii; the Alfvén speed is estimated using a value for the electron density $(N)$ at $26 R$ of $2.37 \cdot 108 \mathrm{~m}^{-3}$ taken from Ulysses radio-ranging experiments (Bird et al., 1996) and magnetic field strength $B_{r}$ (from Ulysses) of $3.5 \mathrm{nT}$ at $430 R$ (Smith et al., 1996). If it is assumed that the solar wind is expanding uniformly at near-constant velocity, then $B_{r}$ and $N$ vary as $R^{-2}$ and the radial profile of the Alfvén speed can be determined. The line inside of 10 solar radii is dashed where the assumptions of constant solar wind velocity and spherical divergence become questionable. The Alfvén wave amplitude was calculated using

$$
\delta V / V_{\text {Alvén }}=\delta B / B_{r} \text {. }
$$

Figure $3 b$ shows the results for Alfvén wave amplitude (bars). Upper bounds derived from angular broadening measurements (Grall et al., 1997) are also shown, represented in the graph by dots and bars, as are curves which represent the evolution of Alfvén wave amplitude according to the WKB approximation (again, assuming spherical divergence and solar wind velocity), with $\delta V$ taken to be $75 \mathrm{~km} / \mathrm{s}$ at the Alfvén critical point, which extrapolates to $\left\langle\delta B^{2}\right\rangle / B_{r}^{2}=1.4$ at $430 R$ ( $2 \mathrm{AU})$. The dashed curve is extrapolated back from Ulysses measurements of Alfvén wave amplitude at $430 R$, where $\left\langle\delta B^{2}\right\rangle / B_{r}^{2}$ was 0.9 (Jokipii et al., 1995). The dot-dash curves are based on estimates of Alfvén wave amplitude high-speed streams as measured by Helios (Roberts, 1989). The minimum and maximum wave dot-dash curves correspond to $\left\langle\delta B^{2}\right\rangle / B_{r}^{2}=1.1$ at $430 R$ (based on the typical value of $\left\langle\delta B^{2}\right\rangle / B_{r}^{2}$ at $1 \mathrm{AU}$ ) and $\left\langle\delta B^{2}\right\rangle / B_{r}^{2}=3.5$ at $430 R$ (based on the maximum wave flux observed by Helios at $65 R$ ).

Klinglesmith (1997) concluded from these results that between 10 and $40 R$ the Alfvén wave evolution was consistent with WKB evolution, thus, reaffirming a previous conclusion by Roberts (1989) that Alfvén waves do not carry enough energy to accelerate the solar wind. The Klinglesmith (1997) conclusion that the wave's amplitude agrees with WKB has low statistical significance, since the data show large scatter: Eq. (1) depends on two main parameters, $R_{0}$ and $\delta V_{0}$, and on many other assumptions (Heinemann and Olbert, 1980; Klinglesmith, 1997) and so the errors in these parameters may be large.

Since these results were presented, a larger data set has become available from observations carried out at EISCAT after 1996. This expanded data set was the main motivation 


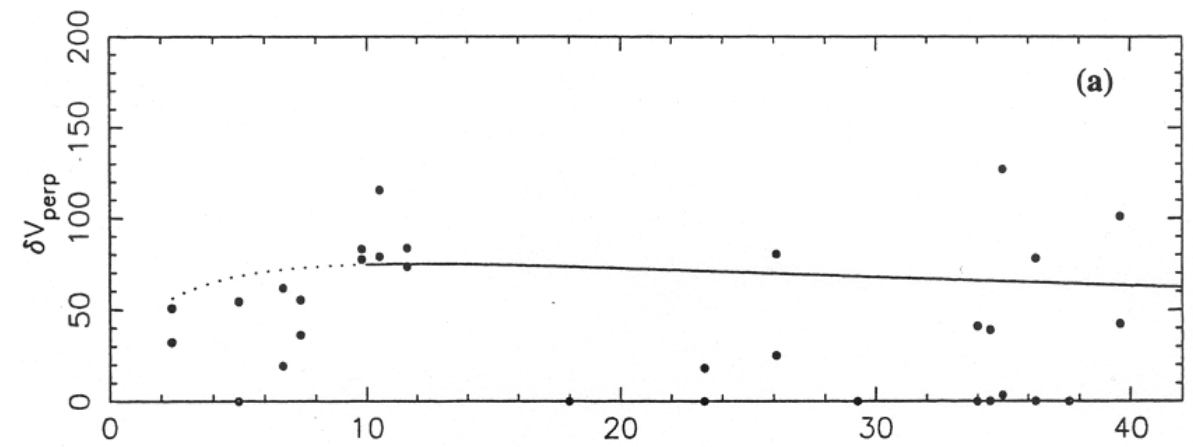

a)

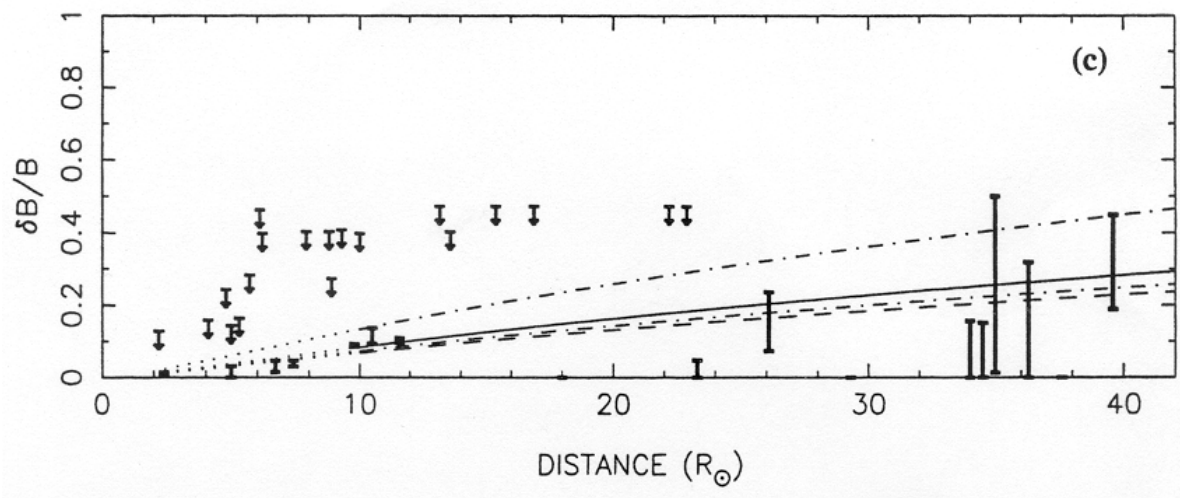

b)

Fig. 3. Results from Klinglesmith (1997) in which (a) RMS- $V_{\perp}-$ referred to as $\delta V_{\perp}$ and (b) Alfvén wave amplitude $-\delta B / B$, are plotted as a function of distance from the Sun.

for the study described here. The expanded data set improves the statistics and extends the coverage of the observations to greater heliocentric distances than was originally the case in the Klinglesmith (1997) study. In addition, the model developed by Ofman and Davila (Ofman et al., 1997; Ofman and Davila, 1997, 1998) proposed that low-frequency (in the millihertz range) large amplitude nonlinear Alfvén waves could transport energy into the acceleration region of the fast solar wind, then dissipate via nonlinear interactions heating the corona and driving the outflow of the fast solar wind. The nonlinear interactions produce compressive fluctuations that can efficiently dissipate via compressive viscosity or Landau damping.

\section{The data set}

In this paper, we present measurements of random transverse velocities made using the European Incoherent Scatter Radar (EISCAT) UHF facility between 1994 and 1999. All the observations used in this study were overwhelmingly dominated by fast flow and, therefore, are predominantly from the years before 1999 when large polar coronal holes were present; the few observations from 1999 are all from high southern latitudes where the large polar fast stream persisted later in the solar cycle (Breen et al., 2002a).
In real observations of the fast solar wind near solar minimum there are normally two components of flow across the ray path - slow flow from above the solar equator near to the plane of the ecliptic (and thus, enveloping the Earth) and fast flow above the polar coronal holes. The relative contributions to the overall scintillation pattern of the fast and slow streams, therefore, depends upon their relative densities, as well as their positions in the ray path. Results presented by Coles et al. (1995) suggested that the slow solar wind was about 4 times denser than the fast wind, which would imply that slow wind streams which lay as far as $60^{\circ}$ from the P-point could still contribute significantly to the overall scattering pattern. More recently, however, an extensive study by Fallows (2001); (Fallows et al., 2002b) has suggested that the difference in density may be smaller, with the slow wind about 2.5 times denser than the fast wind. In this study, the data used were taken from observations for which $\theta_{\text {in }}, \theta_{\text {out }} \geq 35^{\circ}$, i.e. no slow flow was thought to be present within $35^{\circ}$ of the P-point (see Fig. 1 for an illustration of the coordinate system). Unfortunately, only one observation (that of $0738+177$ made on 3 July 1997) had no slow wind within $60^{\circ}$ of the P-point, so it is possible that some of the observations have been affected by slow flow if the Coles et al. (1995) result for the fast and slow wind densities is correct, but since the Fallows et al. (2002b) results are based on EISCAT data from the same period as the observations used 
in this study, we have based our criteria for "fast-dominated" observations on a slow wind 2.5 times denser than the fast wind.

Other criteria in the choice of the data set - some of which were reached during the analysis - ensured that only observations of isolated point sources which could be fitted accurately and could be reproduced were included in the study and that observations which were very noisy or lay in the strong scattering region were rejected. It is most important that only compact sources are used for the study the degree of correlation between the scintillation patterns recorded at the two sites can be significantly reduced if the electric field is not coherent over a transverse scale comparable to the Fresnel scale. For the purposes of IPS observations from EISCAT (centered on 931.5 MHz), a "compact" source should have an angular width of less than 0.06 arcseconds. We have attempted to restrict this study to observations of compact sources, but since the structure of astronomical radio sources is not normally measured at $931.5 \mathrm{MHz}$, some uncertainty remains. We are confident that $0431+$ 206 is sufficiently compact to be regarded as a pure point source in EISCAT observations at $931.5 \mathrm{MHz}$ and believe that the same is true for $0323+055$, but it is possible that some of the other sources used may have some structure which is not clearly resolved in the source-maps produced by interferometers, in which the antennas are separated by distances smaller than the EISCAT baselines. We also restricted the study to sources which were isolated, i.e. there was no other significant scintillating radio source in the antenna beam $\left(0.62^{\circ}\right.$ wide at half-power for the EISCAT antennas). A second, independent compact source would increase the overall level of scintillation recorded (as the scintillations would add), but the scintillation patterns from the two sources would not be correlated, leading to an unrealistically large estimate of random perpendicular velocity from the fitting routines. We have attempted to restrict the study to isolated compact sources, i.e. no other significantly powerful scintillating source lay within two half-power beam widths (1.24 of the source being observed). The criterion adopted in this study for "isolation" was that there should not be any other compact source with a flux strength of more than $0.5 \mathrm{Jy}$ within $1.25^{\circ}$ of the arc of the source observed.

The observations used in this study satisfied all the above criteria and are listed in Table 1.

\section{Initial results and improvements to the analysis tech- nique}

\subsection{Results using a large number of radio sources}

The initial analysis undertaken in this study used a large data set consisting of all low noise, fast stream observations present in the EISCAT IPS database for which stable fits indicating similar values of solar wind parameters were realized by two analysts working separately - in a double blind ap- proach to fitting data. The data included in this first survey had to be

- Fast stream dominated;

- Low noise observations;

- Stable fits, in that repeated fitting at slightly different starting points produced similar final estimates of the final parameters.

The results of this analysis strongly suggested that RMS- $V_{\perp}$ decreased with distance from the Sun and that this was best described by a power-law fit in the form RMS- $V_{\perp} \propto 1 / R$, where $R$ is the distance from the Sun in solar radii. The correlation coefficient of the power-law fit was 0.62 .

\subsection{Improvements in the analysis technique}

In discussions concerning these initial results, it was pointed out by Coles (private communication, 2000) that the outcome of the study might be affected by the de-correlating effect of extended sources. In addition, it was suggested that some of the observations might have been in the strong scattering region where the lower coherence time of the irregularity pattern would have a de-correlating effect. It also became apparent during the analysis that the effects of RMS- $V_{\perp}$ and $\delta V_{\|}$ could not always be simply separated. The final results take this into account. Examination of maps of radio sources and use of the VLA criteria for source size were used to select compact sources, and while some uncertainty remains due to the difference in observing frequencies and baseline lengths between EISCAT and the interferometer networks (the VLA, VLBA and MERLIN) used to construct the maps, we believe that we have restricted the study to compact sources. Careful consideration of the evolution of scintillation strength as sources drifted towards the Sun, as discussed by Fallows et al. (2002b), allowed us to select only observations clearly in weak scattering. Distinguishing between the effects of RMS$V_{\perp}$ and $\delta V_{\|}$required more care, and in the next section, we present 4 methods of estimating these parameters.

It is, in principle, possible that the de-correlation of the scintillation patterns observed at the two IPS sites arises from temporal variations of the irregularity structures in the frame of the solar wind, but if that were the case, we would expect to see a strong dependence between the degree of correlation and the time taken for the irregularity pattern to pass between the two ray paths. The height of the maximum crosscorrelation does decrease as the separation of the ray paths increases (e.g. Moran et al., 1998), though the effect is not very marked, and the observations reported in this paper do not show any substantial dependence on ray path separation. There is likely to be some temporal variation in the scintillation pattern, and this can be modelled as an additional randomly varying velocity. In principle, it might be possible to separate the effects of temporal variation from the transverse velocities introduced by Alfvén waves because temporal variations would be isotropic, whereas RMS- $V_{\perp}$ is, by definition, anisotropic (W. A. Coles, private communication, 
Table 1. EISCAT IPS observations used in this study. The EISCAT IPS data set contained 67 observations of point-like sources which were clearly dominated by fast flow, but not all of these were clearly in the weak scattering region or free of external noise. The 21 observations listed above fulfilled all of these criteria and could be used to obtain robust estimates of RMS- $V_{\perp}$ (repeated fits with different starting points converged to similar values) for at least one of the fitting methods described in Sect. 5.1-5.4

\begin{tabular}{|c|c|c|c|c|c|c|}
\hline Date & Source & Latitude & Longitude & Distance & $\theta_{\text {in }}$ & $\theta_{\text {out }}$ \\
\hline 940429 & $0323+055$ & -54 & 316.1 & 61.5 & -45 & 45 \\
\hline 940430 & $0323+055$ & -56.5 & 302.7 & 59.3 & -60 & 40 \\
\hline 940507 & $0321+123$ & -57.9 & 188.7 & 28.4 & -85 & 35 \\
\hline 940508 & $0321+123$ & -65.5 & 172.6 & 25.9 & -40 & 40 \\
\hline 940511 & $0323+055$ & -76.9 & 265.2 & 46.9 & -40 & 40 \\
\hline 940512 & $0323+055$ & -73.6 & 262.4 & 47.2 & -35 & 55 \\
\hline 940513 & $0323+055$ & -69.9 & 255.6 & 47.8 & -40 & 50 \\
\hline 940514 & $0321+123$ & -64.2 & 257.3 & 23.5 & -35 & 45 \\
\hline 940514 & $0323+055$ & -66.1 & 246.5 & 48.5 & -35 & 65 \\
\hline 940606 & $0431+206$ & 24.7 & -3.5 & 311.6 & -40 & 80 \\
\hline 940607 & $0431+206$ & 28.2 & -2.2 & 298.5 & -40 & 70 \\
\hline 950710 & $0741+312$ & 60.3 & 293.8 & 39.1 & -50 & 65 \\
\hline 950722 & $0741+312$ & 58.1 & 287.2 & 45. & -50 & 55 \\
\hline 960429 & $0323+055$ & -55.2 & 27.6 & 60.4 & -50 & 70 \\
\hline 960502 & $0323+055$ & -63.5 & 349.6 & 54.5 & -50 & 60 \\
\hline 960503 & $0323+055$ & -66.7 & 338.1 & 52.8 & -50 & 75 \\
\hline 970521 & $0432+416$ & 45.1 & 339.8 & 84. & -40 & 70 \\
\hline 970603 & $0432+416$ & 68.8 & 200. & 71.7 & -40 & 90 \\
\hline 970608 & $0432+416$ & 69.4 & 169.9 & 73.6 & -40 & 90 \\
\hline 970703 & $0738+177$ & -23 & 98.8 & 46.2 & -60 & 60 \\
\hline 990508 & $0323+055$ & -79.4 & 253.3 & 47.8 & -45 & 40 \\
\hline
\end{tabular}

2002). However, in this paper, we have assumed that all of the parameters fitted as RMS- $V_{\perp}$ were due to Alfvén waves, in order to establish an upper limit for the transverse velocity of these waves.

\section{Results}

In this section, observations using the following criteria were analyzed. The data had to be from:

- Simple, point like sources;

- Clearly in the weak scattering region;

- Observations overwhelmingly dominated by fast wind;

- Stable fits, in that repeated fitting at slightly different starting points produced similar final estimates of the final parameters;
- Low noise observations.

Of a total of 67 fast wind dominated observations of compact sources made between 1993 and 1999, 21 fulfilled all of these criteria (see Table 1). Most of these observations came from the period near solar minimum in 1995-1997, but observations of the highest southern latitudes continued to be dominated by fast flow into the spring of 1999, as the southern polar fast stream persisted longer in the rise to solar maximum (Breen et al., 2002a; Fallows et al., 2002a).

In order to distinguish between effects of RMS- $V_{\perp}$ and $d V_{\|}$on model fits and to obtain the most robust effects of RMS- $V_{\perp}$, four approaches were used.

\subsection{Fitting both RMS- $V_{\perp}$ and $d V_{\|}$}

For the first set of results both RMS- $V_{\perp}$ and $d V_{\|}$were fitted. The results showed a general trend of RMS- $V_{\perp}$, decreasing 


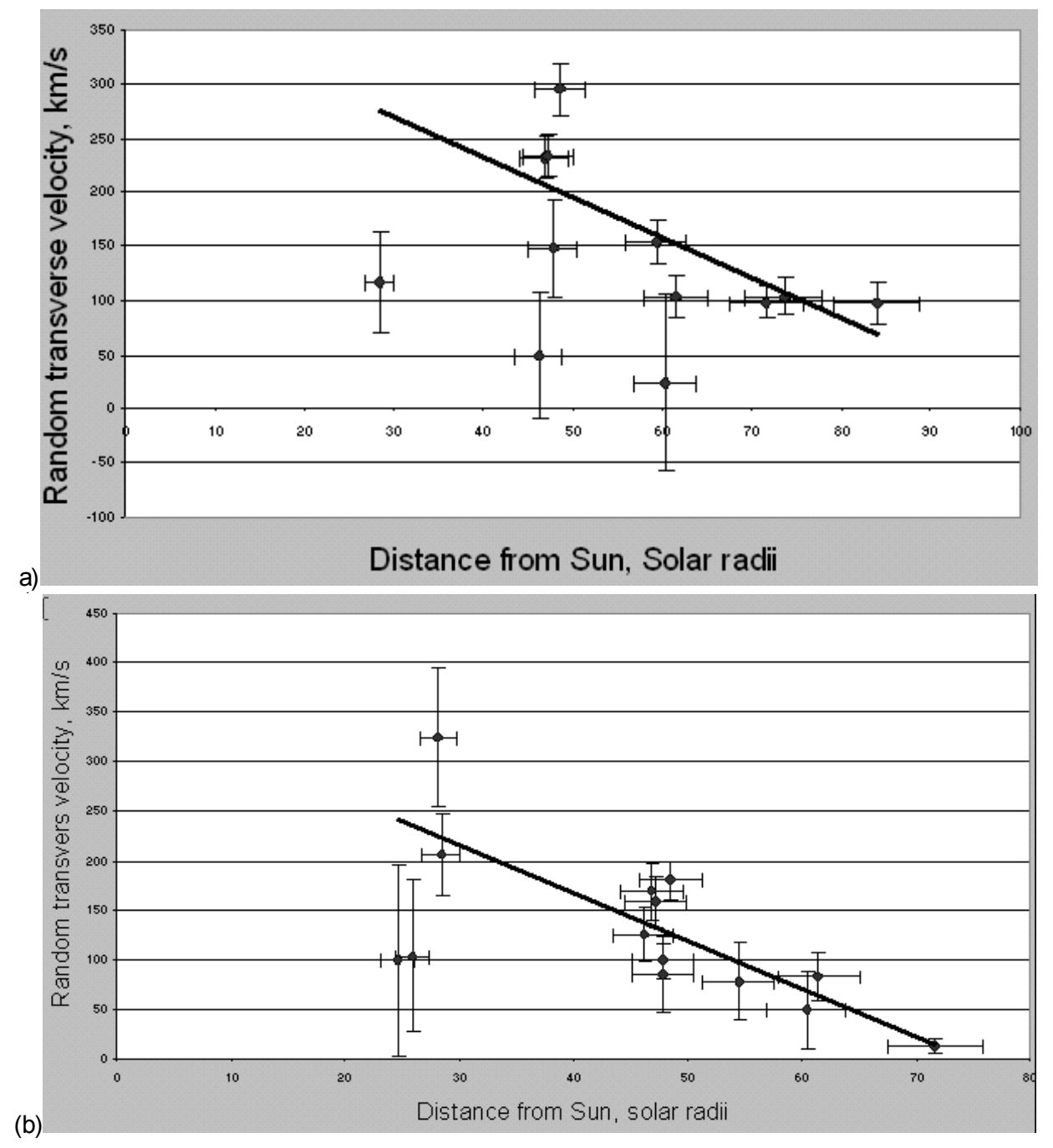

Fig. 4. Random transverse velocity, RMS- $V_{\perp}$ as a function of the P-point distance from the Sun in solar radii $(R)$ when: (a) RMS- $V_{\perp}$ was fitted with $d V_{\|}$held constant at $0 \mathrm{~km} / \mathrm{s}$ (b) RMS- $V_{\perp}$ was fitted with $d V_{\|}$fixed at $15 \%$ of the modelled value for fast bulk flow velocity or $V_{\text {Alvén }} / 2$, whichever is larger.

with distance from the Sun. The correlation coefficient is 0.54 - significant evidence of a linear correlation between RMS- $V_{\perp}$ and distance from the Sun. The probability that 20 measurements of two uncorrelated variables would yield a correlation coefficient $\geq 0.54$ is 0.025 , so these results do suggest a systematic relationship between RMS- $V_{\perp}$ and the distance from the Sun.

\subsection{Fitting RMS- $V_{\perp}$ with $d V_{\|}=0$}

In this study, just RMS- $V_{\perp}$ was fitted and $d V_{\|}$was kept at zero. This is known to be a physically unrealistic assumption, since there will always be a finite variation of outflow velocity over the extended ray path through the solar wind. The results obtained are set out in Table 2, while Fig. 4a shows the fitted values of RMS- $V_{\perp}$ plotted as a function of distance from the Sun. Again, there seems to be a general trend of
RMS- $V_{\perp}$ decreasing with distance from the Sun. However, in this study, just 12 results were obtained and with a correlation coefficient of only 0.34 between the two variables, there is little evidence for any correlation. The probability that 12 measurements of two uncorrelated variables would yield a correlation coefficient of 0.34 is, itself, 0.34 - not indicative of a clear relationship between the derived values of RMS- $V_{\perp}$ and the distance from the Sun. The results of this analysis do, however, provide an ultimate upper bound for RMS- $V_{\perp}$ and are, therefore, an important part of the analysis.

\section{$5.3 \quad$ Fitting RMS- $V_{\perp}$ with $d V_{\|}=0.15 \cdot V_{\text {fast }}$}

Evidence from Ulysses first orbit (Phillips et al., 1994; Woch et al., 1997) showed that fast wind from polar holes at its equatorial boundary had a velocity $12-15 \%$ lower than the 
Table 2. RMS- $V_{\perp}$ values estimated from EISCAT IPS measurements. The results shown in italics are for cases when RMS- $V_{\perp}$ was fitted with $d V_{\|}$fixed at $0 \mathrm{~km} / \mathrm{s}$, while those shown in normal or bold type for cases when RMS- $V_{\perp}$ was fitted with $d V_{\|}$fixed at $15 \%$ of the modelled value for fast bulk flow velocity or $V_{\text {Alvén }} / 2$, whichever is larger. The results shown in bold are those cases in which $d V_{\|}$was set to $V_{\text {Alvén }} / 2$. The distances given are from the center of the Sun to the point of closest approach of the IPS ray path to the Sun (the P-point) and are in units of solar radii. The derived velocity parameters are all in $\mathrm{km} / \mathrm{s}$ described in Sects. 5.1-5.4

\begin{tabular}{|c|c|c|c|c|c|c|}
\hline Date & Source & Distance & RMS- $V_{\perp}$ & $\sigma\left(R M S-V_{\perp}\right)$ & $d V_{\|}$ & $V_{\text {fast }}$ \\
\hline \multirow[t]{2}{*}{940429} & $0323+055$ & 61.5 & 104 & 19 & 0 & 779 \\
\hline & & & 83 & 24 & 110 & 778 \\
\hline 940430 & $0323+055$ & 59.3 & 154 & 19 & 0 & 794 \\
\hline \multirow[t]{2}{*}{940507} & $0321+123$ & 28.4 & 118 & 46 & 0 & 824 \\
\hline & & & 206 & 42 & 158 & 783 \\
\hline 940508 & $0321+123$ & 25.9 & 104 & 77 & 174 & 859 \\
\hline \multirow[t]{2}{*}{940511} & $0323+055$ & 46.9 & 232 & 19 & 0 & 813 \\
\hline & & & 169 & 28 & 120 & 791 \\
\hline \multirow[t]{2}{*}{940512} & $0323+055$ & 47.2 & 234 & 19 & 0 & 816 \\
\hline & & & 158 & 26 & 120 & 792 \\
\hline 940513 & $0323+055$ & 47.8 & 99 & 17 & 102 & 778 \\
\hline \multirow[t]{2}{*}{940514} & $0323+055$ & 48.5 & 295 & 24 & 0 & 809 \\
\hline & & & 180 & 20 & 110 & 811 \\
\hline 940606 & $0431+206$ & 28.2 & 325 & 70 & 182 & 773 \\
\hline 940607 & $0431+206$ & 24.7 & 99 & 97 & 160 & 761 \\
\hline \multirow[t]{2}{*}{960429} & $0323+055$ & 60.4 & 24 & 82 & 9 & 744 \\
\hline & & & 49 & 49 & 129 & 750 \\
\hline 960502 & $0323+055$ & 60.4 & 49 & 40 & 112 & 750 \\
\hline 970521 & $0432+416$ & 84.0 & 97 & 20 & 0 & 732 \\
\hline \multirow[t]{2}{*}{970603} & $0432+416$ & 71.7 & 99 & 15 & 0 & 767 \\
\hline & & & 13 & 7 & 112 & 749 \\
\hline 970608 & $0432+416$ & 73.6 & 104 & 17 & 0 & 781 \\
\hline \multirow[t]{2}{*}{970703} & $0738+177$ & 46.2 & 50 & 58 & 0 & 624 \\
\hline & & & 125 & 28 & 98 & 618 \\
\hline \multirow[t]{2}{*}{990508} & $0323+055$ & 47.8 & 148 & 45 & 0 & 760 \\
\hline & & & 85 & 39 & 112 & 755 \\
\hline
\end{tabular}

velocity over the polar crown. This was consistent with IPS observations (Breen et al., 1996b), which suggested a 12\% difference in velocity between the highest and lowest latitude streams of the fast wind and with coronal and in situ observations reported by Habbal and Woo (2001). Taken together these indicate that a reasonable upper bound for the intrinsic variation of bulk flow speed $\left(d V_{\|}\right)$across a polar coronal hole would be $15 \%$ of the outflow speed. In this way, we have used a priori information from in situ, coronal and IPS measurements to reduce the number of free variables in the model fit. The 13 results showed a decrease in RMS- $V_{\perp}$ with distance from the Sun. The correlation coefficient was 0.76 and since the probability that 13 measurements of two uncorrelated variables would yield a correlation coefficient of 0.76 is 0.008 , this indicates that there is significant evidence of a linear correlation between RMS- $V_{\perp}$ and the distance from the Sun. It is at least interesting that the highest degree of significance of the results obtained occurs when physically realistic assumptions are used to reduce the number of free variables (and the lowest when deliberately unrealistic as- 
sumptions are made, as in Sect. 5.2).

5.4 Fitting RMS- $V_{\perp}$ with $d V_{\|}$set to $0.15 \cdot V_{\text {fast }}$ or $0.5 \cdot V_{\text {Alvén }}$, whichever is greater

IPS observations of the solar wind close to the Sun (Klinglesmith, 1997) have suggested large variations in flow speed (large $d V_{\|}$). Ofman et al. (1997) interpreted this as indicating that, close to the Sun, the density variations giving rise to IPS are moving at a speed different from that of the background plasma, with irregularity speeds varying between $V_{\text {fast }}$ and $V_{\text {fast }}+V_{\text {Alvén }}$, where $V$ is the bulk flow velocity of the fast wind plasma and $V_{\text {Alvén }}$ is the Alfvén velocity. Close to the Sun, the Alfvén speed is large, and $d V_{\|}$will be dominated by the Alfvénic contribution, so that $d V_{\|}=V_{\text {Alvén }} / 2$. Further away from the Sun - outside 30-40 $R$ - intrinsic variations in the solar wind speed will be greater than the Alfvénic contribution and so $d V_{\|}$can still be approximated by $0.15 \cdot V_{\text {fast }}$. In the final section of this study, we took $d V_{\|}$to be $V_{\text {Alvén }} / 2$ or $0.15 \cdot V_{\text {fast }}$, whichever was larger. The results of the analysis are set out in Table 2 and the derived values of RMS- $V_{\perp}$ are plotted as a function of distance from the Sun in Fig. $4 \mathrm{~b}$.

Fourteen observations could be fitted using this method, and the coefficient of correlation between the estimates of RMS- $V_{\perp}$ and the P-point distance was 0.62. The probability that 14 measurements of two uncorrelated variables would yield a correlation coefficient of 0.62 is 0.023 , so although these results are less significant than those obtained in Sect. 5.3, when it was assumed that scintillations drifted at the background flow speed and intrinsic variations in solar wind speed determined $d V_{\|}$, there is still suggestive evidence that RMS- $V_{\perp}$ decreases as the distance from the Sun increases.

Equation (1) describes the variation of the transverse velocity introduced by Alfvén waves as a function of distance from the Sun, provided that the solar wind is expanding radially at constant velocity and is adequately described by the WKB approximation. We have, therefore, fitted Eq. (1) to our data, allowing for the transverse velocity at the critical Alfvén point $\delta V_{0}$ to vary, in order to obtain estimates of these parameters and to test whether they are consistent with those derived from other solar wind measurements and with the current understanding of the fast wind. The results of the model fit are shown in Fig. 5. The agreement between the fitted curves and the estimated values of RMS- $V_{\perp}$ is generally poor for any realistic values of the heliocentric distance of the Alfvén critical point $\left(R_{0}\right)$, without the large values of RMS$V_{\perp}$ (suggested by EISCAT measurements close to the Sun) reflected in the fitted curves. The values of $\delta V_{0}$, suggested by fitting the observed data with $R_{0}=12$, were $85 \mathrm{~km} / \mathrm{s}$ for values of RMS- $V_{\perp}$, determined when $d V_{\|}$was fixed at $0.15 \cdot V_{\text {fast }}$ and $89 \mathrm{~km} / \mathrm{s}$ when $d V_{\|}$was set to the greater of $0.15 \cdot V_{\text {fast }}$ or $0.5 \cdot V_{\text {Alvén }}-$ rather greater than the $75 \mathrm{~km} / \mathrm{s}$ used by Klinglesmith (1997). These estimates of $\delta V_{0}$ should be treated with caution, however, since the fit of the curves to the data is poor.
These results suggest that additional dissipation of lowfrequency Alfvén waves may be taking place in the inner solar wind, as suggested by Ofman et al. (1997) and Ofman and Davila $(1997,1998)$, although the uncertainties in estimates of RMS- $V_{\perp}$ inside $30 R$ are too large for definite conclusions to be drawn.

\section{Conclusions}

The aim of this study was to obtain estimates of randomlyvarying transverse velocity (RMS- $V_{\perp}$ ) in the fast solar wind and, by assuming this component of the velocity to be produced by low-frequency Alfvén waves, to establish experimental constraints on the evolution of the Alfvén wave flux with radial distance and thus, provide a test for models of fast wind acceleration. We have - in the main - succeeded in this aim, although the results close to the Sun are not sufficiently clear-cut to distinguish between the predictions of different acceleration models: estimates of RMS- $V_{\perp}$ have been obtained over a wide range of radial distances and - when physically realistic assumptions are made in the IPS analysis - there is a statistically significant dependence of RMS- $V_{\perp}$ on the distance from the Sun.

Obtaining accurate estimates of RMS- $V_{\perp}$ from IPS data is not easy, and significant improvements in the analysis method had to be made. Even when observations that contain significant external noise have been eliminated, there are still other significant sources of potential error. By restricting the data used to observations of compact, point-like radio sources, the effects of source structure on the observed scintillation pattern could be eliminated, while the scintillation level studies carried out by Fallows et al. (2002b) assisted us in determining which observations lay clearly in weak scattering. The most important advance in the analysis technique, however, has been in the use of a priori information on the latitudinal variation of solar wind velocity (from coronal and in situ measurements, as well as IPS observations) to provide an upper bound for the intrinsic variation in solar wind speed across the fast stream $\left(d V_{\|}\right)$. This reduces the number of free variables in the model fit and allows for RMS$V_{\perp}$ to be determined more reliably. By repeating the model fit with slightly different starting points, the "robustness" of the fit could be tested, and in this study, we have only used the results of model fits which were found to be stable in this way.

The results of this study do suggest that RMS- $V_{\perp}$ decreases as heliocentric distance $(R)$ increases. The degree of correlation and the statistical significance of the relationship both increase when physically realistic assumptions about $d V_{\|}$are made in the model fit, reducing the degree to which the fitting process is unresolved. It is, perhaps, significant that the worst relationship between RMS- $V_{\perp}$ and $R$ was seen when unrealistic assumptions were deliberately made $\left(d V_{\|}=0\right)$.

The decrease in RMS- $V_{\perp}$, with increasing $R$ seen in these results, was not present in the earlier study carried out by 


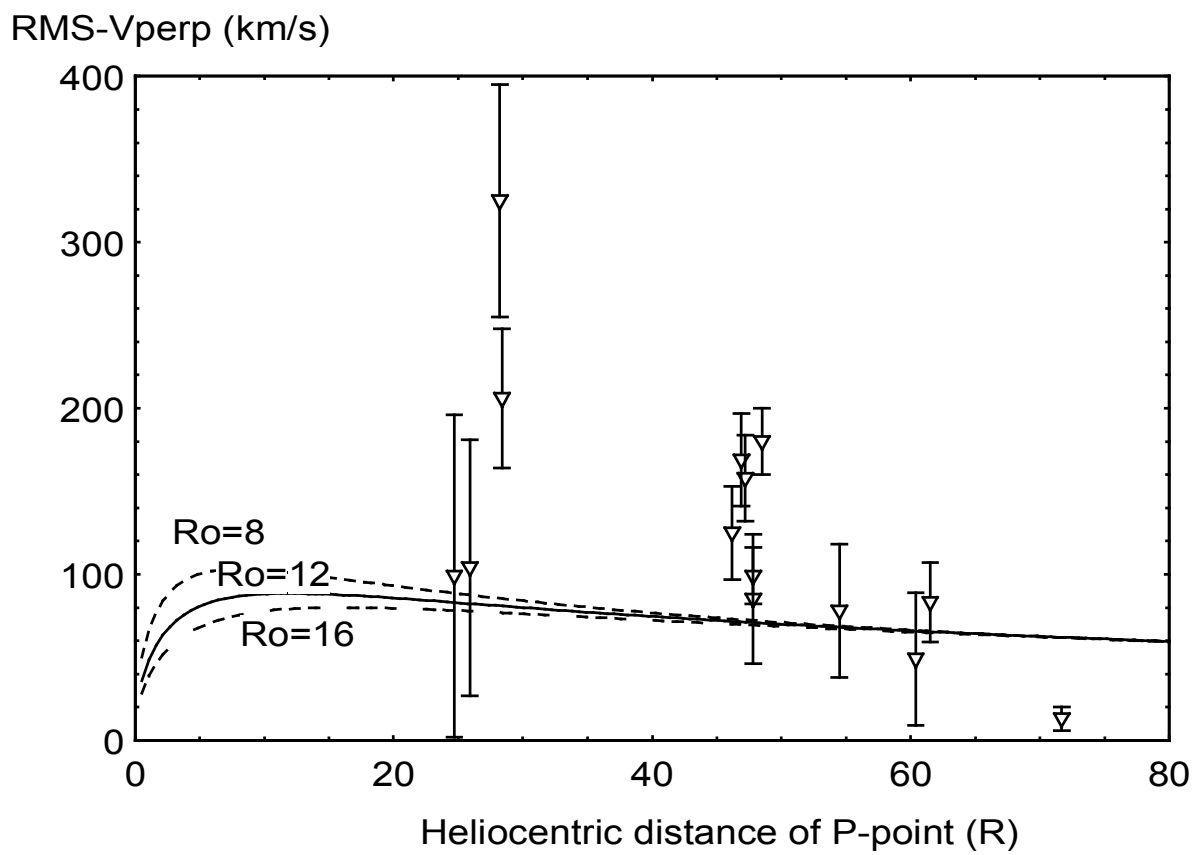

Fig. 5. The three lines show the results of fitting Eq. (1) to the RMS$V_{\perp}$ values derived with $d V_{\|}$fixed at $15 \%$ of the modelled value for fast bulk flow velocity or $V_{\text {Alfvén }} / 2$, whichever is larger. The Alfvén critical distance, $R_{0}$, is taken to be 8,12 and 16 solar radii.
Klinglesmith (1997), which relied on a less comprehensive EISCAT data set, but did include measurements closer to the Sun, made by using the VLBA network. Our results, suggesting significantly larger values of RMS- $V_{\perp}$ in the region, overlap with the Klinglesmith (1997) observations. This, in turn, suggests that the Alfvén waves may carry more energy that can accelerate and heat the solar wind. The larger values of RMS- $V_{\perp}$ closer to the Sun seen in these results do offer some suggestion that the nonlinear effects may be stronger than it appears from previous data and the WKB approximation, in support of the mechanism proposed by Ofman et al. (1997) and Ofman and Davila $(1997,1998)$ for the acceleration and heating of the solar wind. This would be consistent with the difficulty encountered in fitting Eq. (1) (which applies to a WKB wind) to our estimated values of RMS- $V_{\perp}$.

We consider that our results are, at least, suggestive of a relationship between RMS- $V_{\perp}$ and $R$, but the large uncertainties in the RMS- $V_{\perp}$ estimates closest to the Sun and the limited number of observations inside $30 \mathrm{R}$ mean that we cannot determine the form of this relationship, and the results are not wholly inconsistent with those of Ekers and Little (1971) or Klinglesmith (1997), which showed substantial scatter far from the Sun. Measurements closer to the Sun will be required before we can establish the profile of RMS$V_{\perp}$ with radial distance. We hope to extend the EISCAT data set covering $20-80 R$ and to obtain estimates of RMS- $V_{\perp}$ from MERLIN data at $7-10 R$ as soon as sufficiently large fast streams reappear.

Considerable improvements in the IPS analysis technique have resulted from this study, which has produced the most accurate estimates of RMS- $V_{\perp}$ obtained to date. In the future, we hope to extend this study using EISCAT data from the upgraded system and MERLIN data covering regions closer to the Sun. With improvements in computing power, it might be possible to explicitly take into account the structure of the source in the analysis, thereby increasing the number of usable sources. But the uncertainty in source structure at $931.5 \mathrm{MHz}$ when measured with two antennas at separations of 30-400 km (as with EISCAT) makes this impractical at the present time. It may, however, prove possible to adopt this approach when analyzing MERLIN observations as source maps at the same frequency and antenna spacing as the IPS measurements can be obtained by using MERLIN in its primary role as a long-baseline multi-element interferometer.

Acknowledgements. We would like to thank the director and staff of EISCAT for the use of the EISCAT IPS data used in this study. EISCAT is supported by the scientific research councils of Finland, France, Germany, Japan, Norway, Sweden and the UK. Particular thanks are due to A. P. van Eyken for assistance in making EISCAT available for intensive IPS observations.

The IPS analysis routines used in this study were developed at University of California, San Diego and we would like to thank W. A. Coles for making them available to us, as well as for his advice on the minutiae and potential pitfalls of IPS analysis.

Three of us (ARB, PJM and RAF) were supported by PPARC during the period when this work was carried out. AC was funded by the University of Wales, Aberystwyth. LO was supported by NASA SR\&T and Sun Earth Connection Theory programs.

Topical Editor M. Lester thanks W. Coles and A. Hewish for their help in evaluating this paper.

\section{References}

Axford, W. I. and McKenzie, J. F.: The Solar Wind, in: Cosmic Winds and the Heliosphere, (Eds) Jokipii, J. R., Sonnet, C. P., and Giampapa, M. S., pub. University of Arizona Press, 1997. 
Bird, M. K., Petzold, M., Edenhofer, P., Asmar S. W., and McElrath, T. P.: Coronal radio sounding with Ulysses: Solar wind electron density near $0.1 \mathrm{AU}$ during the 1995 conjunction, Astron. Astrophys., 316, 441-448, 1996

Bourgois, G., Coles, W. A., Daign, G., Silen, J., Turenen T., and Williams, P. J. S.: Measurements of solar wind velocity using EISCAT, Astron. Astrophys., 144, 452-462, 1985.

Breen, A. R., Canals, A., Fallows, R. A., Moran P. J., and Kojima, M.: Large-scale structure of the Solar Wind from Interplanetary Scintillation measurements during the rising phase of cycle 23, Adv. Space. Res., 29, 379-388, 2002a.

Breen, A. R., Coles, W. A., Grall, R. R., Klinglesmith, M. T., Markkanen, J., Moran, P. J., Tegid B., and Williams, P. J. S.: EISCAT measurements of the solar wind, Ann. Geophysicae, 14, 1235-1245, 1996b.

Breen, A. R., Coles, W. A., Grall, R. R., Løvhaug, U. P., Markkanen, J., Misawa H., and Williams, P. J. S.: EISCAT measurements of interplanetary scintillation, J. Atmos. Terr. Phys., 58, 507-519, 1996a.

Breen, A. R., de Forest, C. F., Thompson, B. J., McKenzie, J. F., Modigliani, A., Moran, P. J., Varley C. A., and Williams, P. J. S.: Comparisons of interplanetary scintillation and optical measurements of solar wind acceleration with model results, Adv. Space Res., 26, 781-784, 2000.

Breen, A. R., Moran, P. J., Varley, C. A., Wilkinson, W. P., Williams, P. J. S., Coles, W. A., Lecinski A., and Markkanen, J.: Interplanetary scintillation observations of interaction regions in the solar wind, Ann. Geophysicae, 16, 1265-1282, 1998.

Breen, A. R., Thomasson, P., Jordan, C. A., Tappin, S. J., Fallows, R. A., Canals A., and Moran, P. J.: Interplanetary scintillation and optical measurements of slow and fast solar wind acceleration near solar maximum, Adv. Space Res., in press, 2002b.

Coles, W. A.: Interplanetary Scintillation observations of the highlatitude solar wind, Space Sci. Rev., 72, 211-222, 1995.

Coles, W. A.: A bimodal model of the solar wind speed, Astrophys. Space Sci., 243, 87-96, 1996.

Coles, W. A., Esser, R., Løvhaug U.-P., and Markkanen, J.: Comparison of solar wind velocity measurements with a theoretical acceleration model, J. Geophys. Res., 96, 13 849-13 859, 1991.

Coles, W. A., Grall, R. R., Klinglesmith M. T., and Bourgois, G.: Solar cycle changes in the level of compressive microturbulence near the Sun, J. Geophys. Res., 100, 17 069-17 079, 1995.

Coles, W. A., Rickett, B. J., Rumsey, V., Kaufman, J. J., Hurley, D., Ananthakrishnan, S., Armstrong, J., Harmon, J. K., Scott S., and Sime, D.: Solar cycle changes in the polar solar wind, Nature, 286, 239-241, 1980.

Cranmer, S. R.: Ion cyclotron wave dissipation in the solar corona: The summed effect of more than 2000 ion species, Astrophys. J., 532, 1197-1208, 2000.

Dennison, P. A. and Hewish, A.: The solar wind outside the plane of the ecliptic, Nature, 213, 343-346, 1967.

Ekers, R. D. and Little, L. T.: The motion of the solar wind near the Sun, Astron. Astrophys., 10, 310-316, 1971.

Fallows, R. A.: Studies of the solar wind throughout a solar cycle, Ph.D. Thesis, University of Wales, Aberystwyth, 2001.

Fallows, R. A., Breen, A. R., Moran, P. J., Canals A., and Williams, P. J. S.: The high-latitude solar wind in EISCAT IPS data, 19911999, Adv. Space Res., in press, 2002a.

Fallows, R. A., Williams P. J. S., and Breen, A. R.: EISCAT measurements of solar wind velocity and the associated level of interplanetary scintillation, Ann. Geophysicae, this issue, 2002b.

Grall, R. R.: Remote sensing observations of the solar wind near the sun, Ph.D. Thesis, University of California, San Diego, 1995. Grall, R. R., Coles, W. A., Klinglesmith, M. T., Breen, A. R., Williams, P. J. S., Markkanen, J., and Esser, R.: Rapid Acceleration of the polar solar wind, Nature, 379, 429-432, 1996.

Grall, R. R., Coles, W. A., Spangler, S. R., Sakurai, T., and Harmon, J. K.: Observations of field-aligned density microstructure near the Sun, J. Geophys. Res., 102, 263-273, 1997.

Habbal, S. R. and Woo, R.: Connecting the Sun and the solar wind: Comparison of the latitudinal profiles of coronal and Ulysses measurements of the fast wind, Astrophys. J., 549, L253-L256, 2001.

Habbal, S. R., Woo, R., Fineschi, S., O’Neal, R., Kohl, J., Noci, G., and Korendyke, C.: Origins of the slow and the ubiquitous fast solar wind, Astrophys. J., 489, L103-L106, 1997.

Heinemann, M. and Olbert, S.: Non-WKB Alfvén waves in the solar wind, J. Geophys. Res., 85, 1311-1327, 1980.

Hewish, A., Scott P. F., and Willis, D.: Interplanetary Scintillation of small-diameter radio sources, Nature, 203, 1214-1217, 1964.

Jokipii, J. R., Kota, J., Giacalone, J., Horbury, T. S., and Smith, E. J.: Interpretation and consequences of large-scale magnetic variances observed at high heliographic latitude, Geophys. Res. Lett., 22, 3385-3388, 1995.

Klinglesmith, M. T.: The polar solar wind from 2.5 to 40 solar radii: results of intensity scintillation measurements, $\mathrm{Ph}$. D. thesis, University of California, San Diego, 1997.

Kohl, J. L., Noci, G., Antonucci, E., Tondello, G., Huber, M. C. E., Gardner, L. D., Nicolosi, P., Strachan, L., Fineschi, S., Raymond, J. C., Romoli, M., Spadaro, D., Panasyuk, A. V., Siegmund, O. H. W., Benna, C., Cierravella, A., Cranmer, S. R., Giordiano, S., Karovska, M., Martin, R., Michels, J., Modigliani, A., Naletto, G., Pernechele, C., Poletto G., and Smith, P. L.: First results from the SOHO Ultraviolet Coronagraph spectrometer, Sol. Phys., 175, 613-644, 1997.

Kohl, J. L., Noci, G., Antonucci, E., Tondello, G., Huber, M. C. E., Cranmer, S. R., Strachan, L., Panasyuk, A. V., Gardner, L. D., Romoli, M., Fineschi, S., Dobrzycka, D., Raymond, J. C., Nicolosi, P., Siegmund, O. H. W., Spadaro, D., Benna, C., Ciaravella, A., Giordano, S., Habbal, S. R., Karovska, M., Li, X., Martin, R., Michels, J. G., Modigliani, A., Naletto, G., O’Neal, R. H., Pernechele, C., Poletto, G., Smith P. L., and Suleiman, R. M.: UVCS/SOHO Empirical Determinations of Anisotropic Velocity Distributions in the solar corona, Astrophys. J., L501, L127-L131, 1998.

Kojima, M. and Kakinuma, T.: Solar-cycle dependence of global distribution of solar wind speed, Space Sci. Rev., 53, 173-222, 1990.

Krieger, A. S. and Timothy, A. F.: A coronal hole and its identification as the source of a high velocity solar wind stream, Sol. Phys., 29, 505-525, 1973.

Liewer, P., Velli M., and Goldstein, B.: Hybrid simulations of wave propagation and ion cyclotron heating in the expanding solar wind, Space Sci. Rev., 87, 257-260, 1999.

Little, L. T. and Ekers, R. D.: A method of analysing drifting random patterns in astronomy and geophysics, Astron. Astrophys., 10, 306-309, 1971.

Massey, W.: Measuring Intensity Scintillations at the Very Long Baseline Array (VLBA) to probe the solar wind near the Sun, M.Phil. Thesis, University of California, San Diego, 1998.

McComas, D. J., Riley, P., Gosling, J. T., Balogh, A., and Forsyth, R.: Ulysses' rapid crossing of the coronal hole boundary, J. Geophys. Res., 103, 1955-1967, 1998.

McComas, D. J., Barraclough, B. L., Funsten, H. O., Gosling, J. 
T., Santiago-Munoz, E., Skoug, R. M., Goldstein, B. E., Neugebauer, M., Riley, P., and Balogh, A.: Solar wind observations over Ulysses' first polar orbit, J. Geophys. Res., 105, 10419 $10433,2000$.

Moran, P. J., Breen, A. R., Varley, C. A., Williams, P. J. S., Wilkinson W. P., and Markkanen, J.: Measurements of the direction of the solar wind using interplanetary scintillation, Ann. Geophysicae, 16, 1259-1264, 1998.

Neupert, W. M. and Pizzo, V.: Solar coronal holes as sources of recurrent geomagnetic disturbances, J. Geophys. Res., 79, 37013709, 1974.

Nolte, J. T., Krieger, A. S., Timothy, A. F., Gold, R. E., Roelof, E. C., Vaiana, G., Lazarus, A. J., Sullivan, J. D., and McIntosh, P. S.: Coronal holes as sources of solar wind, Sol. Phys., 46, 303-322, 1976.

Ofman, L. and Davila, J. M.: Solar wind Acceleration by solitary waves in coronal holes, Astrophys. J., 476, 357-365, 1997.

Ofman, L. and Davila, J. M.: Solar wind acceleration by large amplitude nonlinear waves: parametric study, J. Geophys. Res., 103, $23677-23690,1998$.

Ofman, L., Davila, J. M., Coles, W. A., Grall R. R., and Klinglesmith, M.: IPS Observations of the solar wind velocity and the acceleration mechanism, The 31st ESLAB Symposium on correlated phenomena at the Sun in the Heliosphere and in Geospace, ESA SP-415, 361-366, ESTEC, Noordwijk, 1997.

Phillips, J. L., Balogh, A., Bame, S. J., Goldsteen, B. E., Gosling, J. T., Hoeksema, J. T., McComus, D. J., Neugebauer, M., Sheeley, N. R., and Yang, Y. M.: Ulysses at $50^{\circ}$ south: Constant immersion in the high speed solar wind, Geophys. Res. Lett., 12, 1105-1108, 1994.
Rickett, B. J. and Coles, W. A.: Evolution of the solar wind structure over a solar cycle: Interplanetary scintillation velocity measurements compared with coronal observations, J. Geophys. Res., 96, 1717-1736, 1991.

Rishbeth, H. and Williams, P. J. S.: Ionospheric Radar: the system and its early results, Mon. Not. R. Astr. Soc., 26, 478-512, 1985.

Roberts, D. A.: Interplanetary observational constraints on Alfvén wave acceleration of the solar wind, J. Geophys. Res., 96, 68996905, 1989

Schwenn, R.: Large-Scale structures of the interplanetary medium, in: Physics of the inner heliosphere 1, (Eds) Schwenn, R. and Marsch, E., Springer, Berlin Heidleberg, New York, 99-181, 1990.

Smith, E. J., Balogh, A., Neugebauer M., and McComus, D.: Ulysses observations of Alfvén waves in the southern and northern hemispheres, Geophys. Res. Lett., 22, 2381-3384, 1996.

Snyder, C. W. and Neugebauer, M.: The relation of Mariner 2 plasma data to solar phenomena, in: The Solar Wind, (Eds) Mackin, R. J. and Neugebauer, M., Pergamon Press, New York, 25-34, 1966.

Tu, C. Y. and Marsch, E.: Wave dissipation by ion cyclotron resonance in the solar corona, Astron. Astrophys., 368, 1071-1076, 2001.

Woch, J., Axford, W. I., Mall, U., Wilken, B., Livi, S., Geiss, J., Gloeckler G., and Forsyth, R. J.: SWICS/Ulysses observations: The three dimensional heliosphere in the declining/minimum phase of the solar cycle, Geophys. Res. Lett., 24, 2885-2888, 1997.

Woo, R. and Martin, J. M.: Source regions of the solar wind, Geophys. Res. Lett., 24, No. 20, 2535-2538, 1997. 